\title{
Developing a Smartphone Application to Support Smoking Behavior Change through Social Comparison*
}

\author{
Christos Maramis, Vasiliki Mylonopoulou, Agnis Stibe, Minna Isomursu, and Ioanna Chouvarda, \\ Member, IEEE
}

\begin{abstract}
The growing field of mHealth has often dealt with the modification of harmful behaviors, such as smoking, that are associated with medical conditions. Smoking behavior has been targeted by numerous mHealth smoking cessation interventions with the help of a wide range of behavior change support (BCS) techniques. However, the exploitation of the established BCS technique of social comparison by mHealth research on smoking cessation has been limited. Based on upto-date BCS theory and following a user-centered design, we have developed a novel smartphone application, namely QuitIT!, for smoking behavior modification with the help of social comparison. This paper presents the development of QuitIT! as well as its preliminary evaluation through a small pilot study. The latter has yield encouraging initial results concerning the feasibility and the effectiveness of QuitIT! as an mHealth tool for smoking BCS.
\end{abstract}

\section{INTRODUCTION}

The field of mHealth has grown over the last decade [1]. During this period, one of the many applications of mHealth has been to support the modification of human behaviors that are associated with a range of medical conditions (e.g., excessive snacking and lack of physical activity with obesity). Within the spectrum of risky or harmful lifestyle behaviors, smoking maintains a prominent position, with proven relations to dozens of diseases [2]. Therefore, it comes as no surprise that smoking monitoring and cessation have been among the most popular mHealth targets. Indeed, dozens of mHealth smoking cessation interventions have been proposed in the scientific literature [3][4], while hundreds of smoking cessation smartphone apps are available in the mobile app stores.

Most smoking cessation interventions, including mHealth apps, view smoking as a harmful behavior that they attempt to modify, based on some underlying theoretical model. A wide range of behavior change support (BCS) techniques has been adopted by such interventions [5]. Persuasive technology comprises technology-oriented BCS approaches [6] and it has used social influence [7] to motivate people in

*Research partially funded by the "Strengthening Post-Doctoral Research" Action of the Greek State Scholarships Foundation (IKY) and the Connected Health Early Stage Researcher Support System (CHESS) project under the Marie Skłodowska-Curie grant with No. 676201.

C. Maramis and I. Chouvarda are with the Dept. of Medicine, Aristotle University of Thessaloniki, Thessaloniki, Greece (phone: +30 2310999247 , e-mail: chmaramis@med.auth.gr, ioannach@auth.gr).

V. Mylonopoulou is with the Interact Research Group, University of Oulu, Oulu, Finland (e-mail: vasiliki.mylo@oulu.fi).

A. Stibe is with the Transforming Wellbeing, ESLSCA Business School Paris, Paris, France (e-mail: agnis@transforms.me).

M. Isomursu is with the Digital Design, IT University of Copenhagen, Copenhagen, Denmark (e-mail: miis@itu.dk). adopting a new behavior. However, the different interconnected aspects of social influence (e.g. social comparison) [8] remain unaddressed most of the times in the designs [9]. Social comparison theory states that, in lack of objective measurements, people compare themselves to others for self-evaluation [10]. In the case of smoking, research has shown that the level of social comparison can act as a predictor of the success of smoking cessation interventions [11]. However, despite the fact that social comparison is widely accepted as a powerful BCS technique [5][12], its exploitation by existing mHealth research on smoking cessation has been limited.

This paper is part of an ongoing research effort to implement a novel smoking cessation mHealth intervention that builds upon a variety of social comparison options. However, the present paper reports on the development of the cornerstone of the aforementioned intervention, i.e., a simple smartphone application named QuitIT! that allows smokers to (1) easily keep track of their daily cigarette consumption over time, and (2) visually compare their consumption with the consumption of other users of the app through a variety of user-selected comparison alternatives. The results from the preliminary evaluation of QuitIT! in a small pilot study are also reported here. The evaluation focuses on the feasibility of the approach but it also provides some first indications of effectiveness for the associated intervention.

\section{DESIGN}

The design of QuitIT! has followed an iterative usercentered design methodology [13]. Usability and software engineering expertise - brought by the UI designer and the developer of the app, respectively - were combined throughout the entire design phase. First, the functionalities of the app were identified, followed by the design of the UI. To this direction, a number of iterations were conducted, consisting of prototyping, evaluating, analyzing and revising steps. As the design iterations progressed, the UI took 3 forms, namely paper prototype, mockups, and functional prototype. In each iteration, the evaluation step was performed by means of the inspection method, i.e., Nielsen's heuristics [14]. Whenever inconsistences between the design and the heuristics were identified, the UI design was revised, initiating a new design iteration. The result is a simple yet modern UI design with minimalistic use of text.

The application of social influence techniques was twofold. The primary employed social influence technique is, of course, the social comparison with respect to the smoking behavior. The users can compare to individual peers, and to simple peer population statistics. Moreover, they can also select between seeing only the comparison of the present date 
and seeing the history of comparison for all past dates. Secondarily, social influence has targeted the compliance of the user to the application, i.e., the cigarette reporting: A daily reminder is presented to the users asking them to report their daily cigarette count while informing them about the amount of people who have already reported counts. By including the number of compliant peers, the reminder motivates the users and promotes social facilitation and normative influence.

\section{DEVELOPMENT}

\section{A. Functionality}

The main functionalities of QuitIT! are outlined below.

User Registration. This functionality is presented the first time QuitIT! is launched and its goal is to collect the required information for registering the user in the QuitIT! platform. The users first enter a unique registration and then the app guides them through the registration process in a wizard-like manner. The information that is collected consists of the users' age, gender, and country of residence; the users also select a unique nickname and a color for the visualization of their own data.

Data Registration. This is one of the two functionalities that are exposed to the user during the main usage of QuitIT! and it becomes available as soon as the registration is completed. The goal of the functionality is to allow the users to easily register the number of cigarettes they smoke daily. Three alternative registration options are supported:

1. Real-time cigarette reporting. The user can report single smoked cigarettes as they are consumed. To report a cigarette in real-time the users just press a floating action button and confirm their intention in the subsequent dialogue. Each reported cigarette is added to the current daily cigarette count.

2. End-of-day cigarette count reporting. The user has also the option to specify the total cigarette count of the present day at once by means of an alert dialogue that is issued every night at a fixed time (22:30). The dialogue also presents the number of cigarettes that have already been reported - if any - to facilitate user recollection.

3. Calendar-based cigarette count reporting. In case the users have failed to use the two previous reporting options for one or more days, the current option where they select a past date on a calendar and enter the corresponding cigarette count. Again, if cigarettes have already been reported for the selected date, the daily count is presented to the user. This option is accessible via the action bar of the main screen of the app.

Data Display. This is the other main usage functionality of QuitIT!, whose purpose is to visualize the cigarette counts of the users and their peers (i.e., other users of the app). This is the main expression of the employed social comparison technique and it is available in the main screen of app. The cigarette counts are visualized on a daily basis as horizontal bars; the bars corresponding to multiple dates forming a vertical cigarette count timeline with the present date on top. The user can change the data display options at will in a dedicated display settings screen that is accessible through the action bar of the main screen; three mutually exclusive peer data options are supported:

1. No peer data. Only the daily cigarette counts of the user at hand are displayed.

2. Aggregate peer data. This option provides daily cigarette count statistics from the entire population of active QuitIT! users. One or more of the minimum, maximum and average daily cigarette count can be selected for display.

3. Individual peer data. This option displays the daily cigarette counts of up to four individual peers. The peers can be either specified by the user using their nicknames or randomly selected by the app.

Data Logging. This functionality is transparent to the app user and its mission is to collect information concerning the interaction of the user with the app, focusing on the elements of the Data Display functionality. These include the visits of the user to the main screen to interact with the displayed data, the revision of the data display options, etc. In all cases, the type of the interaction and its timestamp are logged. The logged data are going to be analyzed offline in order to answer a list of key research questions, such as what is the relative impact of various alternative social comparison options in smoking cessation?

\section{B. User Interfaces}

The user interfaces of QuitIT! are presented in Fig. 1 and Fig. 2 below as screen captures from a running instance of the app. The former figure provides the screens delivering the User Registration functionality, while the latter tackles the two Main Usage functionalities.

\section{Implementation}

QuitIT! has been developed as a native Android app (in Java) using the Android Studio IDE. The app is available on Google Play and it is compatible with Android OS 5.0 or higher. The Alarmmanager and the SyncAdapter services of the Android OS are leveraged for issuing the endof-day reporting alert dialogue and for synchronizing cigarette count and interaction data, respectively. An SQLite database is used for persistent storage. The app is accompanied by a server application that exposes a series of RESTful web services. The server application persistently stores the daily cigarette counts and interaction logs of all the app users and calculates the necessary statistics.

\section{EVALUATION}

\section{A. Pilot Study}

A small-scale pilot study was conducted to test the feasibility of our approach. According to the guidelines of the Human Sciences Ethics Committee of the University of Oulu, the experimental procedure at hand required no statement from the committee. All adult smokers with no comorbidities who are EU citizens were eligible to participate in the pilot study. After a social media based recruitment campaign, 17 volunteers read and signed a digital informed consent explaining the research and the use of their data, which were handled according to the General Data Protection Regulation (GDPR). Among them, 2 subjects withdrawn before the start of the pilot, as they quitted smoking in the meantime. The 
final list of 15 participants includes 9 males and 6 females with a mean age of 32.8 years. The participants came from 5 European countries (Greece, Finland, Portugal, Netherlands, Sweden). For all but one of the participants, QuitIT! has been their first attempt to reduce their cigarette consumption.

The pilot study took place on September and October 2018. The participants used QuitIT! for 30 days to register their own cigarettes and to view the daily cigarette counts of themselves and their peers. A private YouTube playlist with video tutorials on the use of QuitIT! was communicated to the participants one week before the start of the pilot study. The participants were instructed to contact the researchers through email for any technical problems during the study. After the end of the study, interviews with the participants were conducted for feedback collection via teleconferencing.
No major technical issues (e.g., app crashes) were reported by the participants through emails or in the poststudy interviews. However, for 2 of the participants no cigarette count values or interaction logs were uploaded to the server. In essence, those two participants have used QuitIT! in "offline mode", without any social interaction with the other users. Although this issue needs further investigation, it might be caused by internet connectivity problems unrelated to QuitIT!. In total, 214 daily cigarette count entries were stored in the server application. This translates to 0.59 cigarette count entries per participant per day, denoting that by average each participant registered her cigarette count a bit more frequently than once every 2 days. Concerning data logging, 4298 interaction logs were stored in the server, i.e., about 330 interactions per participant.

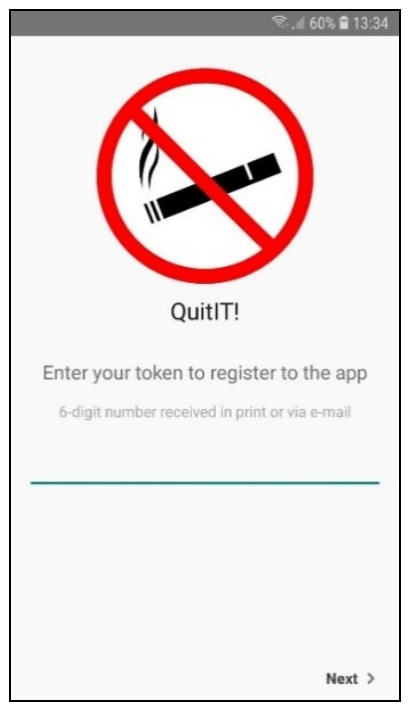

(a)

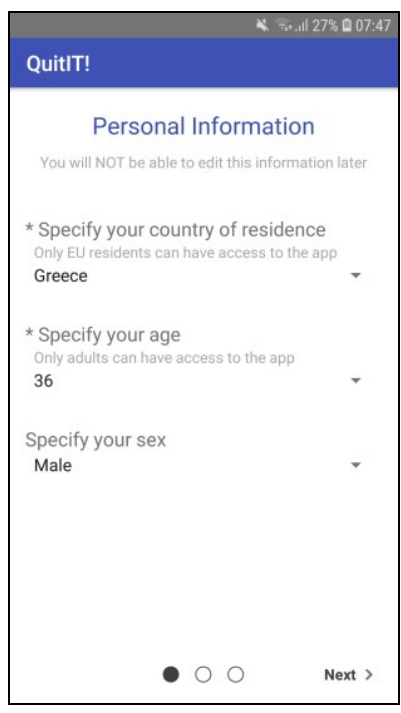

(b)

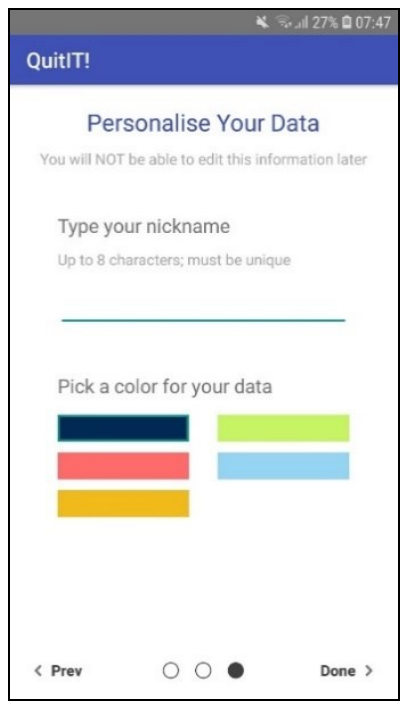

(c)

Figure 1. User Registration Screens: (a) Registration Token Insertion Screen; (b) Personal Data Collection Screen; (c) Personalization Screen.

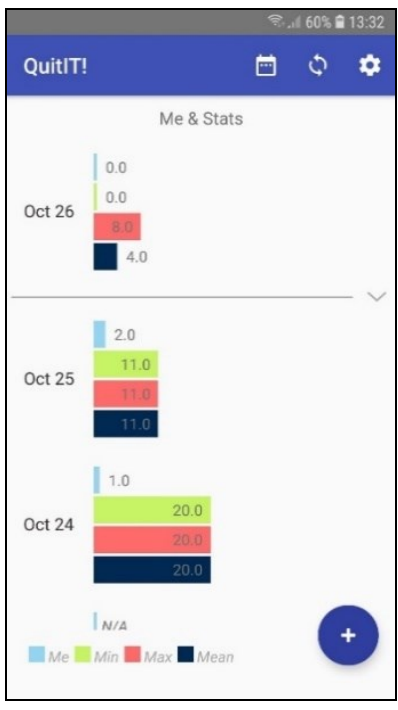

(a)

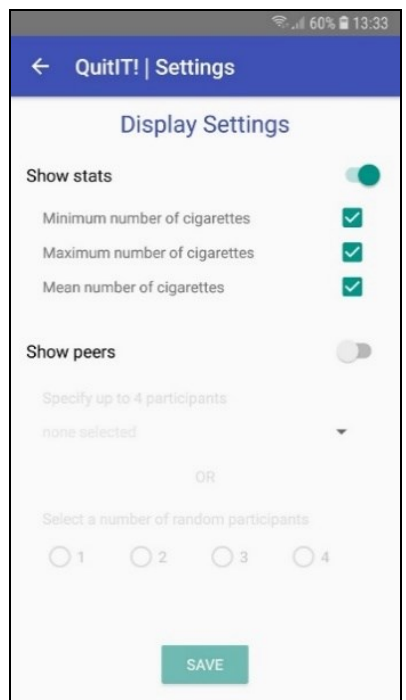

(b)

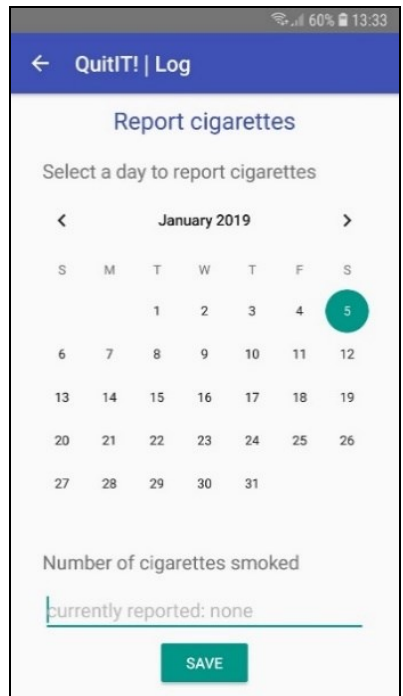

(c)

Figure 2. Main Usage Screens (covers Data Registration \& Data Display): (a) Main Screen; (d) Data Display Setting Screen; (c) Reporting Calendar Screen. 


\section{B. Preliminary Data Analysis}

The dataset that was collected during the pilot study has undergone initial analysis. The preliminary analysis attempted to associate the usage of the app (measured by the days of usage of the app) with the reduction of smoke consumption (measured by the daily cigarette count). The simple - linear correlation of the two variables was tested by calculating the Pearson correlation coefficient (PCC). For an intervention to be effective, which is the end-goal of the present research effort, negative coefficient values need to be reported, i.e., the number of daily cigarettes needs to be reduced over time as the intervention progresses.

Among the 13 participants that synchronized daily cigarette counts with the server application, 2 were excluded from the analysis, the first because she synchronized only 1 daily cigarette count entry and the second because she was not able to use QuitIT! through the entire the pilot study (the smartphone of the participant was stolen during the study). The results of the PCC analysis for the remaining 11 participants are presented in Table 1. Although the number of samples (i.e., daily cigarette count entries) is small to achieve statistical significance, the coefficient value is negative for 7 out of the 11 participants, indicating a tendency to reduce or at least stabilize smoke consumption while QuitIT! is in use.

TABLE I. PCC FOR THE 11 ELIGIBLE STUDY PARTICIPANTS

\begin{tabular}{lrrr}
\hline & User ID & Nr. Samples & PCC \\
\hline $\mathbf{1}$ & 1 & 16 & -0.397 \\
\hline $\mathbf{2}$ & 2 & 30 & -0.144 \\
\hline $\mathbf{3}$ & 3 & 29 & -0.058 \\
\hline $\mathbf{4}$ & 4 & 14 & -0.034 \\
\hline $\mathbf{5}$ & 5 & 20 & 0.110 \\
\hline $\mathbf{6}$ & 8 & 15 & 0.592 \\
\hline $\mathbf{7}$ & 9 & 5 & -0.768 \\
\hline $\mathbf{8}$ & 10 & 24 & 0.028 \\
\hline $\mathbf{9}$ & 14 & 24 & -0.001 \\
\hline $\mathbf{1 0}$ & 15 & 8 & -0.532 \\
\hline $\mathbf{1 1}$ & 16 & 15 & 0.319 \\
\hline
\end{tabular}

\section{DISCUSSION \& CONCLUSION}

The preliminary evaluation of QuitIT! through the conducted pilot study has yield interesting results. Firstly, the usage of the app to carry out the 4 developed functionalities in real-life has proven to be feasible. From the technical standpoint, QuitIT! performed well, demonstrating no cases of major or fatal problems. On top of its robustness, the participants found the app to be easy to use. Most importantly, the initial analysis of the collected dataset has provided some first indications of potential in supporting smoking behavior change. Although the use of the PCC on such a small number of subjects and samples cannot provide any statistically significant results, the fact that over $63 \%$ of the eligible participants shown a decreasing tendency in the number of daily cigarettes is encouraging.
The research on the development of QuitIT! is going to continue. In fact, the participants suggested through their post-study interviews a number of improvements, such as a feature for inviting friends to QuitIT! via personal messages in social media and customizing the timing of the end-of-day reporting alert. The analysis of the interview material is expected to prioritize the suggested improvements. On top of that, the future plans of the authors include the development of features for automatically detecting smoking events [15], offering an appealing alternative to self-reporting with great potential in improving the veracity of the collected data.

The evaluation of QuitIT! should continue as well. Using the pilot study as a guide, large-scale studies involving a much larger number of motivated smokers for a longer period of time need to be organized. The analysis of the large-scale datasets to be collected in these studies is expected to provide concrete evidence about the effectiveness of QuitIT! as an mHealth smoking cessation intervention. Just as importantly, the aforementioned dataset could allow us to quantify the impact of each one of the offered social comparison options (i.e., no peer data, aggregate peer data, and individual peer data) in smoking behavior modification.

\section{REFERENCES}

[1] S. Adibi, Ed., Mobile Health: A Technology Road Map. Springer International Publishing, 2015.

[2] N. J. Wald et al., "Cigarette smoking: an epidemiological overview," Br Med Bull, vol. 52, no. 1, pp. 3-11, Jan. 1996.

[3] R. Whittaker, H. McRobbie, C. Bullen, R. Borland, A. Rodgers, and Y. Gu, "Mobile phone-based interventions for smoking cessation," Cochrane Database of Systematic Reviews, no. 11, 2012.

[4] K. Ghorai, et al., "mHealth for Smoking Cessation Programs: A Systematic Review," J Pers Med, vol. 4, no. 3, pp. 412-423, Jul. 2014.

[5] C. Abraham and S. Michie, "A taxonomy of behavior change techniques used in interventions," Health Psychology, vol. 27, no. 3, pp. 379-387, 2008

[6] B. J. Fogg, "Persuasive technologies," Communications of the ACM, vol. 42, no. 5, pp. 26-27, 1999.

[7] E. Aronson, "The social animal. New York: Worth,", vol. 70, p. 416, 1999.

[8] A. Stibe, "Towards a Framework for Socially Influencing Systems: Meta-analysis of Four PLS-SEM Based Studies," in Persuasive Technology, 2015, pp. 172-183.

[9] V. Mylonopoulou, K. Väyrynen, A. Stibe, and M. Isomursu, "Rationale Behind Socially Influencing Design Choices for Health Behavior Change," in Persuasive Technology, 2018, pp. 147-159.

[10] L. Festinger, "A Theory of Social Comparison Processes," Human Relations, vol. 7, no. 2, pp. 117-140, May 1954.

[11] M. Gerrard, et al., "Smoking cessation: Social comparison level predicts success for adult smokers," Health Psychology, vol. 24, no. 6, pp. 623-629, 2005

[12] H. Oinas-Kukkonen and M. Harjumaa, "Persuasive Systems Design: Key Issues, Process Model, and System Features.," Communications of the Association for Information Systems, vol. 24, 2009.

[13] C. Abras, D. Maloney-Krichmar, et al., "User-centered design," Bainbridge, W. Encyclopedia of Human-Computer Interaction Thousand Oaks: Sage Publications, vol. 37, no. 4, pp. 445-456, 2004.

[14] J. Nielsen, "Iterative user-interface design," Computer, vol. 26, no. 11, pp. 32-41, Nov. 1993.

[15] C. Maramis, et al., "Objective Smoking: Towards Smoking Detection Using Smartwatch Sensors," in Precision Medicine Powered by pHealth and Connected Health, 2018, pp. 211-215. 